\title{
Agiles Arbeiten in offenen \\ Büroumgebungen und Mitarbeiterstress
}

Agilität hat nicht nur in der Softwareentwicklung in der jüngeren Vergangenheit enorm an Bedeutung gewonnen, sondern auch im Zuge der digitalen Transformation und Organisationsgestaltung. Eine höhere Agilität soll in Unternehmen dazu beitragen, rasch auf Veränderungen zu reagieren, um dadurch die Wettbewerbsfähigkeit zu stärken. Viele Organisationen wollen sich in Richtung agiles Unternehmen entwickeln. Eine bedeutsame Eigenschaft des agilen Ansatzes ist das Arbeiten in offenen Büroumgebungen. Man verspricht sich davon verbesserte Kommunikationsprozesse zwischen den Mitarbeitern, die wiederum Einfluss auf die Fähigkeit zur Reaktion auf Veränderungen haben.

\section{René Riedl}


Im vorliegenden Beitrag wird der Frage nachgegangen, ob und in welcher Form das agile Arbeiten in offenen Büroumgebungen den Mitarbeiterstress beeinflusst.

Es wird argumentiert, dass aufgrund vorliegender Forschungsergebnisse davon auszugehen ist, dass agiles Arbeiten in offenen Büroumgebungen beträchtliches Stresspotenzial hat, unter anderem deshalb, weil es eine Unterbrechungskultur befördert. Da Stress nicht nur die Gesundheit und das Wohlbefinden von Mitarbeitern ungünstig beeinflusst, sondern auch betriebswirtschaftliche Kennzahlen wie Leistungsfähigkeit und Produktivität, sollten sich sowohl die Forschung als auch die betriebliche Praxis in Zukunft mehr mit der Gestaltung agiler Arbeitswelten unter expliziter Berücksichtigung einer Stressperspektive auseinandersetzen.

Dem Begriff „Agilität“ kann man sich im digitalen Zeitalter nicht entziehen. Man vernimmt ihn im Kontext der digitalen Transformation und Organisationsgestaltung sowie im Zusammenhang mit Softwareentwicklung. Der Begriff stammt vom lateinischen Wort „agilitas“ ab, was wörtlich übersetzt „Beweglichkeit“ bedeutet; der Begriff ,agilis“ bedeutet "gewandt“. Mit „agil sein“ meint man somit „beweglich sein“ und „gewandt sein“. Es wird prognostiziert, dass in Zukunft viele Unternehmen nach agilen Methoden arbeiten werden, insbesondere deshalb, weil man damit - im Vergleich zu traditionellen Arbeitsweisen - besser auf sich verändernde Anforderungen reagieren kann, was zu einer Stärkung der Wettbewerbsfähigkeit führt [1].

Bedeutsame Charakteristika einer agilen Arbeitsweise sind: kundenzentriert sein, Produkte und Dienstleistungen iterativ entwickeln, ein mitarbeiterzentriertes Führungsverständnis haben, sich weiterentwickeln und innovativ sein, offen gegenüber anderen sein und Netzwerke bilden, aus Fehlern lernen, offen gegenüber unkonventionellen Arbeitsformen sein sowie transparent und stetig dialogbereit sein [2]. Insbesondere die beiden letztgenannten Charakteristika führen in der praktischen Umsetzung zu einer Ausbreitung neuer Bürokonzepte. Wesentliches Merkmal solcher Bürolandschaften ist eine offene Raumfläche, die die Zusammenarbeit und Kommunikation unter den Beschäftigten unterstützen soll. Konzepte wie nichtterritoriales Arbeiten (die fixe Zuordnung von Arbeitsplätzen auf bestimmte Mitarbeiter wird aufgehoben, auch als Flex-Büros bezeichnet) und Co-Working-Spaces (Vermietung von Arbeitsplätzen in offenen Raumumgebungen) spielen hierbei eine große Rolle [3, 4].

Offene Raumflächen können Vorteile in der Zusammenarbeit bringen und somit die wirtschaftliche Schlagkraft ei-

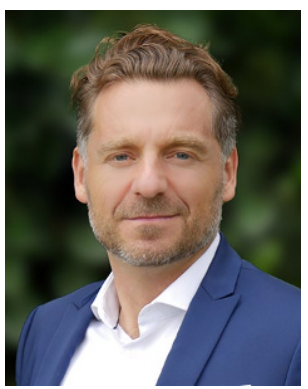

Prof. Dr. René Riedl ${ }^{1,2}(\bowtie)$ ist seit 2013 Inhaber einer Professur für Digital Business und Innovation an der Fachhochschule Oberösterreich, Fakultät für Wirtschaft und Management (Campus Steyr), und dort aktuell Vizedekan und für Forschung und Entwicklung zuständig; zudem ist er Assoziierter Universitätsprofessor am Institut für Wirtschaftsinformatik - Information Engineering an der Johannes Kepler Universität (JKU) Linz. Weiter ist er der wissenschaftliche Direktor der NeuroIS Society (http://www.neurois. org/) und Mitglied des Vorstands am Institut für Personal- und Organisationsentwicklung in Wirtschaft und Verwaltung an der JKU Linz. rene.riedl@fh-steyr.at

\section{${ }^{1}$ University of Applied Sciences Upper} Austria, Wels, Österreich

${ }^{2}$ Johannes Kepler Universität (JKU) Linz, Linz, Österreich

nes Unternehmens erhöhen [3]. Eine Thematik, mit der man sich bislang im Diskurs zur Agilität jedoch kaum befasst hat, ist das Stresspotenzial, das von offenen Raumflächen ausgehen kann. Dies ist problematisch, denn Befunde empirischer Forschung zeigen, dass das Stresspotenzial hoch sein kann. Unternehmen setzen aktuell in teilweise recht unreflektierter Weise offene Raumflächenkonzepte im Zuge ihrer Agilitätsbemühungen um. Konsequenz ist, dass man eigentlich für die Organisation Positives bewirken will, nämlich agiler zu werden, gleichzeitig aber die damit einhergehenden weiteren Effekte wie erhöhten Mitarbeiterstress und dessen Konsequenzen wie Rückgang von Mitarbeitergesundheit und 


\section{Zusammenfassung}

- Agiles Arbeiten in offenen Büroumgebungen gewinnt in der Praxis an Bedeutung.

- Oft bleiben dabei jedoch die negativen Wirkungen unberücksichtigt.

- Mitarbeiterstress ist eine der wichtigsten negativen Konsequenzen offener Büroumgebungen, weil er Gesundheit, Wohlbefinden und wirtschaftliche Leistungsfähigkeit ungünstig beeinflusst.

Wohlbefinden sowie Reduktion von Leistungsfähigkeit und Produktivität außer Acht lässt.

Im vorliegenden Beitrag werden vor dem Hintergrund dieser aktuellen Entwicklung Erkenntnisse wissenschaftlicher Studien beschrieben, die Aufschluss über das Stresspotenzial offener Bürokonzepte geben. Ziel der Abhandlung ist es, Entscheidungsträgern in der Praxis „die Augen zu öffnen“. Bei Gestaltungsentscheidungen im Zuge der Transformation zu einer agilen Organisation sollten Forschungserkenntnisse über Zusammenhänge und kausale Wirkmechanismen berücksichtigt werden, anstatt „blind“ Moden zu folgen.

\section{Studien zu agiler Softwareentwicklung und Stress}

In der wissenschaftlichen Forschung hat man sich bislang bei Anwendung agiler Methoden auf Stress aus Entwicklerperspektive fokussiert. Es werden nachfolgend kompakt wichtige Befunde zu Entwicklerstress zusammengefasst.

Auf der Basis einer Onlinebefragung unter 466 Entwicklern in einem finnischen Technologiekonzern, in dem zum Zeitpunkt der Befragung ein mehrjähriger Übergang von klassischer Entwicklung auf Scrum und Kanban stattfand, zeigte sich folgendes Bild auf die Frage „Wie ist Ihr Stress-Level nach dem Übergang auf agil?": 27 \% besser, 34 \% gleich, $15 \%$ schlechter, $23 \%$ gaben an, dass ihrer Auffassung nach zwischen agilen Methoden und Stress kein Zusammenhang besteht [5]. In einer weiteren Untersuchung in drei finnischen Unternehmen mit insgesamt 44 Teilnehmern wird darüber berichtet, dass agiles Arbeiten Stress reduzieren kann („,results provide evidence that agile work could even out workload during a working period“) [6]. Schließlich zeigen Befragungsdaten aus der Schweiz von 185 IT-Mitarbeitern aus dem agilen Umfeld (vorwiegend Softwareentwickler, Designer und Projektmanager), dass auf einer Skala von 1 (entspannt) bis
5 (sehr gestresst) der mit Abstand dominierende Wert 3 ist; der Stress wird somit vorwiegend als mittelmäßig ausgeprägt wahrgenommen [7]. Interessant ist hierbei, dass die Stresswerte mit den Werten auf die Frage nach dem Agilitätslevel (von $1=$ mehrheitlich planbasiert bis $5=$ mehrheitlich agil) kaum korrelierten. Dies legt nahe, dass zwischen dem Grad der Verwendung agiler Methoden und dem Stress eines ITMitarbeiters bestenfalls ein geringer Zusammenhang besteht.

In der Gesamtschau zeigen die vorliegenden Ergebnisse somit kein eindeutiges Bild. Es ist zukünftig noch viel an Forschung nötig, um herauszufinden, ob und in welcher Weise das Entwickeln nach agilen Methoden den Stress von Softwareentwicklern und anderen IT-Mitarbeitern beeinflusst. Zu betonen ist, dass die bisherige Forschung auf die Untersuchung von Entwicklern fokussierte und somit Benutzer- bzw. Mitarbeiterstress nicht im Fokus stand. Schließlich ist es bedeutsam, anzuführen, dass in den bisherigen Arbeiten nicht explizit auf das mögliche Stresspotenzial offener Büroflächen eingegangen wurde. Dies ist verwunderlich, sind sie doch ein wesentliches Merkmal agiler Arbeitsumgebungen, unabhängig davon, ob es sich um Agilität im Zusammenhang mit Softwareentwicklung handelt oder um Agilität als allgemeines Prinzip im Kontext der digitalen Transformation und Organisationsgestaltung.

\section{Agiles Arbeiten und Mitarbeiterstress}

Es gibt Argumente, warum sich Mitarbeiterstress beim Einsatz agiler Methoden erhöhen könnte. Das Arbeiten in Teams, eine wesentliche Eigenschaft des agilen Ansatzes, geht mit potenziellen Risiken einher, von denen etliche zu Stress führen können. Beispiele sind: höhere Wahrscheinlichkeit für Konflikte, schlechtere Zuordnung von Verantwortung bei Fehlern und Missständen, Bildung von rivalisierenden Teilgruppen, Gefahr divergierender Einstellungen und Verhaltensweisen sowie Gefahr unproduktiver Gruppendiskussionen [1]. Zudem erhöht sich beim Einsatz agiler Verfahren der Kommunikations- und Koordinationsaufwand, wodurch Unterbrechungen der Mitarbeiter wahrscheinlicher werden. Oftmalige Unterbrechungen wirken stresserhöhend [8]. Weiter hat die Forschung gezeigt, dass im Falle einer etablierten Kultur selbstorganisierender Teams der soziale Druck, seinen individuellen Beitrag im Team zu leisten, sehr hoch sein kann, was wiederum mit Stress einhergeht [9].

\section{Büroraumgestaltung und Mitarbeiterstress}

Die Etablierung agiler Organisationsformen geht mit einer Tendenz zu offenen Bürokonzepten einher. Wissenschaftli- 
che Studien der letzten Jahrzehnte zeigen, dass das Arbeiten in Großraumbüros und offen gestalteten Büros erhöhten Mitarbeiterstress bewirken kann. Eine experimentelle Studie aus den USA belegt, dass das Arbeiten in offenen und somit relativ lauten Büroumgebungen zu Anstiegen des Stresshormons Adrenalin und zu Motivationsrückgängen bei der Arbeitserledigung führen kann [10]. Zudem zeigt dieses Experiment, dass das Arbeiten in vergleichsweise lauten Büroumgebungen bewirkte, dass Mitarbeiter mit einer geringeren Wahrscheinlichkeit ergonomische und haltungsmäßige Anpassungen in Computer-Interaktionssituationen machen. Dies ist ein Risikofaktor für die Entwicklung von Haltungsschäden. Bei der Interpretation dieser Befunde ist zu beachten, dass bei zwei weiteren Stresshormonen (Noradrenalin, Kortisol) sowie bei wahrgenommenem Stress (Fragebogenerhebung) keine signifikanten Unterschiede zwischen der lauten offenen Büroumgebung und einer leiseren Umgebung festgestellt werden konnten.

In einer Fallstudie in einem kanadischen Unternehmen wurden Mitarbeiter beim Umstieg von traditionellen Büroumgebungen auf eine offene Büroumgebung untersucht [11]. „Traditionell“ bedeutete mehrheitlich Einzelbüros, rund $13 \mathrm{~m}^{2}$ groß, mit einem Schreibtisch und einer Tür zum Gang. „Offen" bedeutete hingegen mit bis zu neun Personen auf nebeneinander liegenden Arbeitsplätzen. Es gab drei Zeitpunkte, zu denen eine Befragung der Mitarbeiter stattfand: Zeitpunkt t1 unmittelbar vor dem Umstieg, t2 vier Wochen nach dem Umstieg und Zeitpunkt t3 sechs Monate nach dem Umstieg. Folgende Faktoren wurden erhoben: 1. Zufriedenheit mit der physischen Umgebung (zum Beispiel genügend Ablageflächen), 2. Wahrnehmungen zum Stress in der Büroumgebung

\section{Befunde empirischer Studien}

- Offene Büroumgebungen führten zu Anstiegen des Stresshormons Adrenalin und zu Motivationsrückgängen bei der Arbeitserledigung.

- Auch sechs Monate nach einem Umstieg auf eine offene Büroumgebung waren die Mitarbeiterzufriedenheit, der wahrgenommene Stress und die wahrgenommene Arbeitsleistung ungünstig verändert (im Vergleich zur Situation vor dem Umstieg).

- Oftmalige Unterbrechungen bei der Erledigung von Arbeitsaufgaben und eine beeinträchtige Privatsphäre sind wichtige Ursachen von Stress in offenen Arbeitsumgebungen.

\section{Handlungsempfehlungen}

- Unternehmen sollten im Zuge ihrer Agilitätsbemühungen nicht ohne systematische Wirkungsanalyse und somit unreflektiert auf offene Büroumgebungen umstellen.

- Insbesondere die Stresswirkungen in der Belegschaft sollten untersucht werden, da Mitarbeiterstress mit vielen weiteren negativen individuellen (z. B. Gesundheit) und betrieblichen (z. B. Produktivitätsrückgänge) Wirkungen einhergeht.

- Meinungen betroffener Mitarbeiter zu offenen Raumkonzepten sollten vorab eingeholt werden, wenn neue Arbeitsplatzformen in Planung sind.

(Lichtverhältnisse, Temperatur, Belüftung, Umgebungslärm durch Telefongespräche sowie Unterhaltungen von Kollegen), 3. Zufriedenheit mit den Beziehungen zu Teammitgliedern und 4. wahrgenommene Arbeitsleistung (zum Beispiel in der Lage sein, auf die Arbeitsaufgaben fokussiert zu sein und die geplanten Aufgaben ordnungsgemäß zu erledigen). Die Studienergebnisse belegen, dass sich mit dem Wechsel auf offenere Büroumgebungen alle vier Faktoren zum Negativen entwickelten. Dieser negative Effekt fiel auch nach sechs Monaten nicht ab, sondern stieg weiter an bzw. stagnierte. Daraus folgt, dass die im Zeitpunkt t2, also vier Wochen nach dem Wechsel festgestellten negativen Auswirkungen weniger die Konsequenz des Umstiegs an und für sich sind, sondern eher längerfristig anhaltende Negativentwicklungen.

Die meisten Menschen, mit denen der Verfasser in den vergangenen Jahren in der Praxis gesprochen hat, berichten über mehrheitlich negative Erfahrungen beim Arbeiten in offenen Büroumgebungen. Neben den oftmaligen Unterbrechungen und die dadurch ausgelöste Störung der Konzentration auf die Arbeitsaufgabe wird angegeben, dass auch die Privatsphäre in offenen Umgebungen leidet $[12,13]$. Insbesondere gilt dies, wenn Mitarbeiter aus höheren Positionen im Unternehmen, die im Regelfall Einzelbüros gewohnt sind, in offene Büroumgebungen wechseln müssen [14]. Die Ergebnisse einer Erhebung aus dem Jahr 2019 in Deutschland mit über 1000 Befragten zeigen, dass private Gespräche mit Kollegen, die Geräuschkulisse von Kollegen und das Smartphone die Top-3-Ablenkungsfaktoren im Arbeitsumfeld sind [15]. Bezeichnend ist, dass alle drei Faktoren insbesondere in offenen Büroumgebungen ihre negativen Wirkungen voll entfalten. 
In einer bereits in den 1970er-Jahren von Wissenschaftlern des Massachusetts Institute of Technology (MIT) durchgeführten Untersuchung wurde festgestellt, dass eine als „nicht territorial“ designte Büroumgebung zwar die Kommunikation unter den Mitarbeitern signifikant erhöhte, jedoch zu keiner messbaren Steigerung der Abteilungsleistung führte [16]. Was in dieser jahrzehntelang zurückliegenden Studie als „nicht territorial" bezeichnet wurde, ist mit den heute vielerorts anzutreffenden modernen Büroumgebungen beim agilen Arbeiten unmittelbar vergleichbar. Man findet im Bürobereich verteilte Tische und Arbeitsplätze vor, die keinem Mitarbeiter zugeordnet sind. Die Angestellten haben somit keinen fixen Arbeitsplatz und wechseln ihren Schreibtisch nicht nur an verschiedenen Arbeitstagen, sondern oft auch mehrfach an bestimmten Tagen (Flex-Büros). Ein Ende 2019 im Harvard Business Review veröffentlichter Beitrag mit dem Titel „The Truth About Open Offices" bestätigt die MIT-Forscher [17]. Auf der Basis von Fallstudien wird unter anderem berichtet, dass offene Raumkonzepte zu weniger sinnvoller Interaktion zwischen den Mitarbeitern führten. Zudem wird angegeben, dass Unternehmen bereits wieder von offen gestalteten Büros abkehren und auf kleinere Büros zurückwechseln.

Herauszuheben ist der Befund der MIT-Studie, dass mehr Kommunikation nicht notwendigerweise mit mehr Leistung einhergeht. Dies sollte zu denken geben. Gerade in Zeiten ständiger Unterbrechungen durch Instant Messenger, E-Mails und weitere Technologien ist es wichtig, Arbeitsumgebungen zu schaffen, in denen man ungestört einer Tätigkeit nachgehen kann. Die heute vielerorts anzutreffenden offenen Büroumgebungen lassen ein ungestörtes Arbeiten jedoch kaum zu. Das erklärt, warum viele Mitarbeiter heutzutage in offenen Büroumgebungen Kopfhörer tragen. Sie wollen so zumindest den vielen akustischen Ablenkungen entkommen. Doch was bleibt noch von der Hauptmotivation des Schaffens offener

\section{Internet}

Die Website www.digital-stress.info informiert über Forschung zu digitalem Stress und erprobten Bewältigungsstrategien sowie über das im Herbst 2020 erschienene Buch „Digitaler Stress: Wie er uns kaputt macht und was wir dagegen tun können“" (René Riedl, Linde International). Bei digitalem Stress handelt es sich um eine Stressform, die durch die Nutzung und Allgegenwärtigkeit von digitalen Technologien entsteht.
Büroumgebungen, nämlich der gesteigerten Kommunikation, wenn Mitarbeiter wie „Legehennen mit Kopfhörer“ in offenen Büroumgebungen sitzen? Wahrscheinlich wenig. Es verwundert daher nicht, dass in aktuellen Werken zur Gestaltung agiler Arbeitsumgebungen über „Denkerzellen“ und „schallgedämmte Telefonkabinen" berichtet wird, in denen man konzentriert und unter Wahrung von Vertraulichkeit seinen Tätigkeiten nachgehen kann [3]. Vielleicht ist es daher doch so, wie man hier und da vernimmt, dass unter dem Deckmantel „gesteigerter Kommunikation im agilen Zeitalter" Einzelbüros oder Büros mit wenigen Mitarbeitern aufgelöst und Großraumbüros bzw. offene Arbeitsumgebungen geschaffen werden, obwohl das primäre Ziel die Senkung von Kosten ist. Es ist bekannt, dass langfristig die Unterhaltung vieler kleiner Büros teurer ist als das Betreiben von Großraumbüros [18].

Eine weitere mögliche Erklärung für die aktuell zunehmende Verbreitung von offenen Bürokonzepten ist, dass dies „en vogue" ist. Man will sich modern geben. So zu sein, wie die vielen Start-ups im Silicon Valley oder sonst wo auf dieser Welt und wie die „Internet-Giganten“ Google oder Facebook, kann verlockend sein. Der Verfasser kennt jedoch keine Quelle, die wissenschaftlich nachweist, dass der Erfolg dieser Unternehmen seine Ursache in den dort vorzufindenden Großraumbüros und offenen Büroflächen hat. Viele Menschen ziehen falsche Schlüsse, wenn sie einerseits Bilder von den „coolen“ Büroumgebungen sehen und andererseits lesen, wie erfolgreich die Unternehmen sind. Man ist verleitet, die Ursache des Unternehmenserfolgs in den offenen Büroumgebungen zu sehen. Solange nicht gegenteilige Evidenz vorgelegt wurde, sollte man sich daher nicht verleiten lassen, falsche Schlüsse zu ziehen.

\section{Fazit}

Ist das Arbeiten in offenen Büroumgebungen so wie es zunehmend öfter bei agiler Arbeitsplatzgestaltung vorkommt eine Stressquelle für Mitarbeiter? Auf der Basis der hier präsentierten Forschungsbefunde ist zu konstatieren, dass dies naheliegend ist. Mit dem Einsatz agiler Methoden können viele Chancen einhergehen [1]. Insbesondere sind dies betriebswirtschaftliche Vorteile wie rascheres Reagieren auf Marktanforderungen. Der Einsatz agiler Verfahren ist daher in vielen Situationen berechtigt. Verantwortungsträger in Unternehmen sollten aber beachten, dass sich der Mitarbeiterstress beim Einsatz agiler Methoden erhöhen kann. Die Schaffung von stressreduzierenden Arbeitsumgebungen muss daher ein wichtiges Ziel in jedem Unternehmen sein. Es ist zu fordern, dass Unternehmen bei der Transformation zur agilen Orga- 
nisation und der damit einhergehenden Etablierung offener Büroumgebungen explizit den damit verbundenen Mitarbeiterstress im Fokus haben. In aktuellen Schriften zur Gestaltung agiler Arbeitswelten wird nicht ausreichend bzw. oft gar nicht auf Befunde wissenschaftlicher Forschung zu den Stress-, Zufriedenheits- und Leistungswirkungen offener Büroumgebungen eingegangen. Es bleibt zu hoffen, dass dieser Artikel dazu beiträgt, die Diskussion und Forschung in diesem Bereich in Gang zu setzen.

Funding. Open access funding provided by University of Applied Sciences Upper Austria.

Open Access Dieser Artikel wird unter der Creative Commons Namensnennung 4.0 International Lizenz veröffentlicht, welche die Nutzung, Vervielfältigung, Bearbeitung, Verbreitung und Wiedergabe in jeglichem Medium und Format erlaubt, sofern Sie den/die ursprünglichen Autor(en) und die Quelle ordnungsgemäß nennen, einen Link zur Creative Commons Lizenz beifügen und angeben, ob Änderungen vorgenommen wurden.

Die in diesem Artikel enthaltenen Bilder und sonstiges Drittmaterial unterliegen ebenfalls der genannten Creative Commons Lizenz, sofern sich aus der Abbildungslegende nichts anderes ergibt. Sofern das betreffende Material nicht unter der genannten Creative Commons Lizenz steht und die betreffende Handlung nicht nach gesetzlichen Vorschriften erlaubt ist, ist für die oben aufgeführten Weiterverwendungen des Materials die Einwilligung des jeweiligen Rechteinhabers einzuholen.

Weitere Details zur Lizenz entnehmen Sie bitte der Lizenzinformation auf http://creativecommons.org/licenses/by/4.0/ deed.de

\section{Literatur}

[1] Riedl, R. (2019). Management von Informatik-Projekten: Digitale Transformation erfolgreich gestalten (2. Aufl.). München: De Gruyter Oldenbourg. Lerneinheit „Agile Methoden in InformatikProjekten“"

[2] Conboy, K. (2009). Agility from first principles: reconstructing the concept of agility in information systems development. Information Systems Research, 20(3), 329-354.

[3] Klaffke, M. (2019). Gestaltung agiler Arbeitswelten: Innovative Bürokonzepte für das Arbeiten in digitalen Zeiten. Wiesbaden: Springer.

[4] Fincke, I., et al. (2018). Arbeitsbedingungen in innovativen Bürokonzepten unter gesundheitsrelevanten und arbeitswissenschaft- lichen Aspekten. Zentralblatt für Arbeitsmedizin, Arbeitsschutz und Ergonomie, 3, 168-176.

[5] Laanti, M. (2013). Agile and wellbeing: stress, empowerment, and performance in Scrum and Kanban teams. In Proceedings of Hawaii International Conference on System Sciences (S. 4761-4770).

[6] Tuomivaara, S., et al. (2017). Short-term physiological strain and recovery among employees working with agile and lean methods in software and embedded ICT systems. International Journal of Human-Computer Interaction, 33(11), 857-867.

[7] Meier, A. (2018). Stress in agile software development: practices and outcomes. In J. Garbajosa, et al. (Hrsg.), Agile processes in software engineering and extreme programming (S. 259-266). Cham: Springer.

[8] Galluch, P., et al. (2015). Interrupting the workplace: examining stressors in an information technology context. Journal of the Association for Information Systems, 16(1), 1-47.

[9] Barker, J. R. (1993). Tightening the iron cage: concertive control in self-managing teams. Administrative Science Quarterly, 38(3), 408-437.

[10] Evans, G. W., \& Johnson, D. (2000). Stress and open-office noise. Journal of Applied Psychology, 85(5), 779-783.

[11] Brennan, A., et al. (2002). Traditional versus open office design: a longitudinal field study. Environment and Behavior, 34(3), 279-299.

[12] Hedge, A. (1982). The open-plan office, a systematic investigation of employee reaction to their work environment. Environment and Behavior, 14(5), 519-542.

[13] Brookes, M. J., \& Kaplan, A. (1972). The office environment: space planning and affective behavior. Human Factors, 14(5), 373-391.

[14] Zalesny, M., \& Farace, R. (1987). Traditional versus open offices: a comparison of socio technical, social relations, and symbolic meaning perspectives. Academy of Management Journal, 30(2), 240-259.

[15] YouGov (2019). Fast jeder ist am Arbeitsplatz abgelenkt. https://de.statista.com/infografik/17433/fast-jeder-ist-am-arbeitsplatz-abgelenkt/. Zugegriffen: 1. Mai 2020.

[16] Allen, T. J., \& Gerstberger, P. G. (1973). A field experiment to improve communications in a product engineering department: the nonterritorial office. Human Factors, 15(5), 487-498.

[17] Bernstein, E., \& Waber, B. (2019). The truth about open offices. Harvard Business Review, 6, 83-91.

[18] Kamarulzaman, N., et al. (2011). An overview of the influence of physical office environments towards employee. Procedia Engineering, 20, 262-268.

Springer Professional $\mid \begin{aligned} & \text { Mehr zum Thema finden Sie online } \\ & \text { www.springerprofessional.de/wum }\end{aligned}$ 